\title{
Association of a Variant of CNR1 Gene Encoding Cannabinoid Receptor 1 With Gilles de la Tourette Syndrome
}

\author{
Natalia Szejko ${ }^{1,2 \dagger}$, Jakub Piotr Fichna ${ }^{3 \dagger}$, Krzysztof Safranow $^{4}$, Tomasz Dziuba ${ }^{1}$, \\ Cezary Żekanowski ${ }^{3}$ and Piotr Janik ${ }^{1 * t}$
}

OPEN ACCESS

Edited by:

Fabricio A. Pamplona,

Entourage Phytolab, Brazil

Reviewed by:

Stefan Unterecker,

University Hospital Würzburg,

Germany

Geison Souza Izídio,

Federal University of Santa Catarina,

Brazil

Daniel Luís,

HeartGenetics, Genetics and Biotechnology, S.A.,

Portugal

*Correspondence:

Piotr Janik

piotr.janik@wum.edu.pl

${ }^{t}$ These authors have contributed equally to this work

Specialty section:

This article was submitted to Behavioral and Psychiatric Genetics,

a section of the journal

Frontiers in Genetics

Received: 23 December 2018 Accepted: 03 February 2020

Published: 04 March 2020

Citation:

Szejko N, Fichna JP, Safranow K, Dziuba T, Żekanowski $C$ and Janik P (2020) Association of a Variant of CNR1 Gene Encoding Cannabinoid Receptor 1 With Gilles de la Tourette Syndrome. Front. Genet. 11:125 doi: 10.3389/fgene.2020.00125
1 Department of Neurology, Medical University of Warsaw, Warsaw, Poland, 2 Department of Bioethics, Medical University of Warsaw, Warsaw, Poland, ${ }^{3}$ Laboratory of Neurogenetics, Department of Neurodegenerative Disorders, Mossakowski Medical Research Centre, Polish Academy of Sciences, Warsaw, Poland, ${ }^{4}$ Department of Biochemistry and Medical Chemistry, Pomeranian Medical University, Szczecin, Poland

Background: Gilles de la Tourette syndrome (GTS) is a neuropsychiatric disorder of unknown etiology, although a major role of genetic factors has been established. Cannabis-based medicines may alleviate GTS-associated tics and variants of CNR1 gene encoding central cannabinoid receptor (CB1) are believed to be a risk factor for the development of some neurodevelopmental diseases. Our aim was to test the association of selected CNR1 gene variants with GTS.

Material and Methods: The cohort of GTS cases comprised 262 unrelated patients aged 3-53 years (mean age: $18.3 \pm 9.1$ years; 204 males (77.9\%), $126(48.1 \%)$ adults defined as $\geq 18$ years). As a control group we enrolled 279 unrelated, ethnically and gender matched individuals with no diagnosed mental, neurological or general disorder, aged 1354 years (mean age: $22.5 \pm 3.0$ years; 200 males, (74.1\%). Both study and control groups were selected from Polish population, which is ethnically homogenous subgroup of Caucasian population. Four single nucleotide polymorphisms (SNPS) in CNR1 were selected: rs2023239, rs2180619, rs806379, and rs1049353 based on minor allele frequency in general population $>15 \%$. These variants were genotyped using a realtime quantitative polymerase chain reaction system (TaqMan SNP genotyping assay).

Results: We found significant association of GTS clinical phenotype with rs2023239 variant. Minor allele $\mathrm{C}$ and $\mathrm{CT}+\mathrm{CC}$ genotypes were found significantly more often in GTS patients compared to controls (17.4 vs $11.1 \%, p=0.003$ and 32.8 vs $20.4 \%, p=0.001$, respectively), and the difference remained significant after correction for multiple testing. C allele of rs2023239 polymorphism of the CNR1 gene was associated with the occurrence of tics. There were no statistically significant associations for rs806379, rs1049353 or rs2180619 variants.

Conclusion: Our findings suggest that $\mathrm{C}$ allele of rs2023239 polymorphism of the CNR1 gene is a risk factor of GTS in Polish population. The variant can be potentially associated 
with abnormal endocannabinoid transmission, which is suspected to be one of the causes of GTS.

Keywords: Gilles de la Tourette syndrome, endocannabinoids, association study, CNR1 gene, CB1cannabinoid receptor 1

\section{INTRODUCTION}

Gilles de la Tourette syndrome (GTS) is neuropsychiatric disorder in which motor and vocal tics occur. They are usually accompanied by psychiatric comorbidities such as attentiondeficit hyperactivity disorder (ADHD), obsessive-compulsive disorder (OCD), depression or self-injury. Segregation analysis in GTS-risk families suggests a complex, polygenic mode of inheritance involving various loci (Mcmahon et al., 1996). Susceptibility genes associated with GTS include: Slit and Trklikel (SLITRK1), L-histidine decarboxylase (HDC), mitochondrial inner membrane protease subunit 2 (IMMP2L), neuroligin 4, X-linked (NLGN4X), contactin-associated protein 2 (CNTNAP2) (for details consult review by Paschou et. al (Paschou et al., 2014), cadherin EGF LAG seven-pass G-type receptor 3 (CELSR3) (Willsey et al., 2017; Wang et al., 2018), WW and $\mathrm{C} 2$ domain containing 1 (WWC1), Nipped-B-like (NIPBL), fibronectin 1 (FN1) (Willsey et al., 2017), arylacetamide deacetylase (AADAC) (Yuan et al., 2018) and others.

Abnormal dopaminergic neurotransmission in the cortex and in the basal ganglia interacting with other neuronal pathways, including endocannabinoid system (ECS), seems to be crucial in GTS pathophysiology (Martino and Leckman, 2013). Endocannabinoids mediate two pathways in the basal ganglia: neurons of the direct pathway expressing dynorphin and D1 dopamine receptor, as well as the indirect pathway neurons expressing enkephalin and D2 receptor (Steiner and Gerfen, 1998; Mechoulam and Parker, 2013). There are many further indications for an involvement of ECS in GTS (Sandyk, 1988; Leckman et al., 1988; Haber and Wolfer, 1992; Muller-Vahl, 1998; Muller-Vahl, 2002; Müller-Vahl et al., 2002; Hasan et al., 2010; Brunnauer et al., 2011; Mechoulam and Parker et al., 2013; Trainor et al., 2016; Jakubovski and Müller-Vahl, 2017; Kanaan et al., 2017). Some post-mortem studies support cannabinoid dysregulation in GTS, as decreased immunoreactivity with dynorphin A [1-17] has been detected in the striatal area of GTS patients (Haber and Wolfer, 1992). Furthermore, increased levels of dynorphin A [1-8] were found in the cerebrospinal fluid of GTS patients which correlated with OCD severity (Leckman et al., 1988). The ECS involvement in GTS is also indicated by a neuroimaging study using single photon emission computed tomography (SPECT) with [ $\left.{ }^{123} \mathrm{I}\right] \mathrm{AM} 281$ (Berding et al., 2004). The clinical efficacy of cannabis-based medicines (CBM) also supports the involvement of ECS (Müller-Vahl et al., 2002; Müller-Vahl et al., 2003). Additionally, a number of retrospective reports on self-medication with CBM (Hemming and Yellowlees, 1993; Muller-Vahl, 2002; Brunnauer et al., 2011; Jakubovski and Müller-Vahl, 2017) as well as case reports (Muller-Vahl, 2002; Hasan et al., 2010; Brunnauer et al., 2011;
Trainor et al., 2016; Jakubovski and Müller-Vahl, 2017; Kanaan et al., 2017) suggest effectiveness of CBM in GTS.

The two main cannabinoid receptors are $\mathrm{CB} 1$ and $\mathrm{CB} 2$, cannabinoid receptors type 1 and 2. CB1 receptor is mostly present in the central nervous system, with a high density in basal ganglia (Pertwee, 2006), the brain region implicated in movement control, while CB2 receptor is mainly present in the immune system and in hematopoietic cells (Russo, 2013) where it modulates cytokine release (Suárez-Pinilla et al., 2015). Variants of the CNR1 gene encoding CB1 receptor have been associated with various psychiatric and neurological diseases (Gadzicki et al., 1999; Gadzicki et al., 2004; Domschke et al., 2008; Evans et al., 2016; Ruiz-Contreras et al., 2017; Yao et al., 2018) (see Table 1). Gadzicki et al. (1999) were the first to investigate polymorphisms in a coding exon of the CNR1 gene finding the $1359(\mathrm{G} / \mathrm{A})$ polymorphism in German GTS patients. Posteriorly they focused on CNR1 variants in a larger group of GTS patients (Janik et al., 2014). However, they failed to find a correlation between those variants and GTS.

Despite that negative outcome we decided to revise the question of a possible role of $\mathrm{CB} 1$ receptor in GTS. We focused on the $\mathrm{CB} 1$ receptor as it is mainly expressed in the brain and mediates inhibition of dopaminergic, glutaminergic, and GABAergic neurotransmission in CNS (Haughey et al., 2008). We hypothesized that ECS dysregulation in GTS could be related to some minor variants of the CNR1 gene and

TABLE 1 | SNPs selected for the study.

\begin{tabular}{|c|c|c|c|}
\hline SNP & $\begin{array}{l}\text { MAF } \\
\text { (NFE) }\end{array}$ & Localization & Associated neuropsychiatric disorders \\
\hline rs2023239 & $16 \%$ & intron & $\begin{array}{l}\text { substance dependence (Haughey et al., } \\
\text { 2008; Filbey et al., 2010), eating disorders } \\
\text { (Müller et al., 2007), schizophrenia (Yu et al. } \\
\text { 2013), impulsivity (Ehlers et al., 2007), } \\
\text { depression (lcick et al., 2015), cyclic } \\
\text { vomiting syndrome (Wasilewski et al., 2017) }\end{array}$ \\
\hline rs2180619 & $40 \%$ & promoter & $\begin{array}{l}\text { anxiety (Lazary et al., 2009), substance } \\
\text { dependence (Chen et al., 2008), eating } \\
\text { disorders (Müller et al., 2008), personality } \\
\text { disorders (Yao et al., 2018) }\end{array}$ \\
\hline rs806379 & $47 \%$ & intron & $\begin{array}{l}\text { anxiety (Lester et al., 2017), substance } \\
\text { dependence (Evans et al., 2016), } \\
\text { schizophrenia (Yu et al., 2013), impulsivity } \\
\text { (Buchmann et al., 2015) }\end{array}$ \\
\hline rs1049353 & $27 \%$ & $\begin{array}{l}\text { exon } \\
\text { (synonymous) }\end{array}$ & $\begin{array}{l}\text { substance dependence (Hindocha et al., } \\
\text { 2019), eating disorders (Sadeghian et al., } \\
\text { 2018), schizophrenia (Suárez-Pinilla et al., } \\
\text { 2015), depression (Mitjans et al., 2013), } \\
\text { multiple sclerosis (Varadé et al., 2012) }\end{array}$ \\
\hline
\end{tabular}

$M A F$, minor allele frequency, in gnomAD database; associated neuropsychiatric disorders based on SNPedia database.

SNP, single nucleotide polymorphism. 
therefore tested four such variants as possible risk factors of GTS in Polish GTS patients.

\section{MATERIAL AND METHODS}

\section{Ethics Statement}

The study has been approved by the Ethics Committee of the Medical University of Warsaw (KB/2/2007, KB/53/A/2010, KB/ $63 / A / 2018$ ) and has therefore been performed in accordance with the ethical standards laid down in the 1964 Declaration of Helsinki and its later amendments. All participants aged $\geq 18$ years signed an informed consent form prior to their inclusion in the study, and legal guardians gave a written consent on behalf of individuals under 18 .

\section{Study Participants}

The study group comprised 262 unrelated GTS patients aged 353 years (mean age: $18.3 \pm 9.1$ years; 204 males $(77.9 \%), 126$ (48.1\%) adults defined as $\geq 18$ years). The family history of tics was positive in 142 (54.8\%) patients, and was unknown for three patient. The family history of OCD or obsessive-compulsive behavior (OCB) was available from 125 patients, and was positive for $37(29.6 \%)$ patients. The mean age of tic onset was $6.9 \pm 3.0$ years. Two hundred and three $(77.8 \%)$ patients had at least one of the following co-morbidities: OCD/OCB $n=127$ (48.7\%), ADHD $\mathrm{n}=90$ (34.5\%), non-OCD anxiety disorder $\mathrm{n}=84(32.2 \%)$, learning disorder $\mathrm{n}=64(24.5 \%)$, depression $\mathrm{n}=$ $37(14.2 \%)$, conduct disorder $\mathrm{n}=16(6.1 \%)$, while $58(22.2 \%)$ had none of the above. Yale Global Tic Severity Scale (YGTSS) was used to assess the severity of tics, but could only be performed for $127(48.5 \%)$ patients. The control group comprised 279 unrelated, ethnically and gender matched individuals with no diagnosed mental, neurological or general disorders, aged 13-54 years (mean age: $22.5 \pm 3.0$ years; 200 males, (74.1\%). The age of the controls was slightly higher (mean age of the two groups differed only by four years) as we wanted to be sure that the controls have passed the age of tic onset (the onset of GTS after 13 years is very rare).

The patients were evaluated for the clinical diagnosis of GTS and co-morbid mental disorders according to DSM-IV-TR, most of them before the DSM-5 criteria were published. Patients who fulfilled the DSM-5, but not DSM-IV-TR diagnostic criteria for GTS were excluded to ensure homogeneity of group. OCB was diagnosed if obsessions and compulsions were egosyntonic in contrast to egodystonic symptoms which characterized OCD. The diagnosis of co-morbid mental disorders was also made based on earlier psychiatric examinations that had been performed before the time of patients' evaluation. This included psychiatric disorders that were usually diagnosed in the childhood (e.g., attention deficit hyperactivity disorder or oppositional defiant disorder) and symptoms of which were not yet present in adult patients at the time of examination. All the patients were referred to a neurologist experienced in tic disorders and were personally interviewed by an author of the study (PJ). The study was designed as a one-time registration study and no new clinical data obtained on follow-up visits have been included in the analysis.

\section{Genetic Analysis and Selection of SNPs}

DNA samples were collected between 2007 and 2018. Genomic DNA was extracted either from peripheral blood leukocytes using a standard salting-out procedure (Miller et al., 1988) or from buccal cells collected with Oragene OG-500 DNA collection kit and using Prep IT L2P purification kit (DNA Genotek Inc., Ottawa, Ontario, Canada). The latter method was used with young patients that were unable or unwilling to comply with blood collections. DNA samples obtained with both methods were of same quality and applicability. Genotyping of selected SNPs was performed using TaqMan SNP genotyping assays (Life Technologies, Carlsbad, California, USA) on a StepOne Plus Real-Time PCR system (Life Technologies) (Janik et al., 2014).

The rs2023239, rs2180619, rs806379, and rs1049353 CNR1 polymorphisms were chosen on the basis of their minor allele frequency (MAF) estimated above 15\% in both general and Polish populations, and their well-documented association with various neurological and psychiatric disorders (Table 1). As indicated by data from the Exome Aggregation Consortium (ExAC) and the Genome Aggregation Database (GnomAD) rs1049353 is the only known polymorphism in the coding sequence of the CNR1 gene with a MAF above 1\%; it is synonymous. All other published variants in the coding sequence are private withfrequency of at most hundreds per 276,000 alleles (MAF below 0.4\%) and therefore were not selected for the study. Also in our in-house database of whole exome/genome sequencing results for $>200$ Polish patients not affected by GTS, the rs1049353 variant is the only one located in the coding sequence of CNR1. Thus, except for rs1049353, the selected SNPs are located in non-coding sequences. For clarity we note that rs1049353 is different from the $1359(\mathrm{G} / \mathrm{A})$ polymorphism studied by Gadzicki et al. (Mackie, 2006).

\section{Statistical Analysis}

Chi-square test was used for both allelic and genotypic association studies. Association of genotypes with age of tic onset and YGTSS was analyzed with Kruskal-Wallis test. Multivariate logistic regression model was used to find independent predictors of GTS risk. The study sample size was sufficient to detect with $80 \%$ probability the true effect size measured as odds ratio (OR) between 1.62 and 1.97 for positive association or between 0.39 and 0.61 for negative association (depending on the actual MAF which ranged from 0.11 to 0.47 ) for the differences in allele frequencies of the four polymorphisms between the GTS and the control group. Statistica 13 program was used for statistical calculations. The significance level was set at $\mathrm{p}<0.05$, and Bonferroni-corrected significance criterion was used where indicated in the text.

\section{RESULTS}

The genotyping success rate was $100 \%$ and the consensus rate (on the basis of $10 \%$ duplicates) was $100 \%$ for DNA isolated from 
both leukocytes and buccal cells. The quality of genotyping was the same regardless of the biological source and method used for DNA isolation. The genotype frequencies of all four SNPs were in accordance with the Hardy-Weinberg equilibrium in the control group ( $\mathrm{p}>0.9$ for $\mathrm{rs} 806379, \mathrm{p}>0.3$ for $\mathrm{rs} 2023239, \mathrm{p}>0.6$ for rs2180619 and $\mathrm{p}>0.6$ for rs1049353). In the patient group the genotype frequencies of three SNPs were in accordance with the Hardy-Weinberg equilibrium ( $p>0.2$ for $\mathrm{rs} 2023239, \mathrm{p}>0.1$ for rs2180619 and p>0.2 for rs1049353) while the distribution of the rs806379 genotype deviated slightly from the Hardy-Weinberg equilibrium ( $\mathrm{p}=0.047)$ due to a deficit of heterozygotes.

A significant association with the GTS was found for the rs2023239 variant. The MAF of CNR1 rs2023239 was significantly higher in GTS patients compared to control group. When the TT genotype was used as a reference, genotypes CC+CT (dominant model, $\mathrm{p}=0.001$ ) and CT alone $(\mathrm{p}=0.0009)$ were significantly associated with a higher risk of GTS. This association remained significant after Bonferroni correction for multiple tests (Bonferroni-corrected significance level for four SNPs and six genetic models is $\left.0.05 /\left(4^{\star} 6\right)=0.002\right)$. There were no statistically significant associations between the rs2180619, rs806379 rs1049353 variants and the GTS (Table 2). Multivariate logistic regression analysis adjusted for gender and age showed that the presence of CNR1 rs2023239 allele C (genotypes $\mathrm{CC}+\mathrm{CT}$, dominant model) is an independent factor associated with a higher risk of GTS (Table 3).
Genotypic and allelic association analysis of all four CNR1 polymorphisms with most common co-morbid disorders in patients with GTS are shown in Table 4. The only significant association was found for rs806379. The minor allele $\mathrm{T}$ of this SNP was more frequent in GTS patients with ADHD than in the patients without ADHD (OR 1.45, 95\% CI: 1.01-2.08, p=0.045). No associations were found between the CNR1 genotype and age of tic onset $(p>0.4)$, total YGTSS or YGTSS Total Tic Score ( $p>0.15)$, family history of tics $(p>0.15)$ or family history of OCB/OCD $(\mathrm{p}>0.2)$.

\section{DISCUSSION}

So far the only comprehensive study investigating genetic variation in the CNR1 gene in GTS (Janik et al., 2014) was aimed to localize pathogenic mutations in CNR1 and failed to

TABLE 3 | Multivariate logistic regression analysis of independent factors associated with presence of GTS as dependent variable (GTS patients vs controls).

\begin{tabular}{lcccc}
\hline Independent variables & OR & \multicolumn{2}{c}{$\mathbf{9 5 \%} \mathbf{C l}$} & $\mathbf{p}$ \\
\hline Gender (male vs female) & 0.922 & 0.601 & 1.414 & 0.710 \\
Age (per 1 year) & 0.906 & 0.879 & 0.934 & $<0.00001$ \\
CNR1 rs2023239 (CC+CT vs TT) & 1.725 & 1.146 & 2.598 & 0.009
\end{tabular}

TABLE 2 | Genotype and allele distribution of the selected SNPs in patients with Gilles de la Tourette syndrome and controls.

\begin{tabular}{|c|c|c|c|c|c|c|c|c|c|c|}
\hline \multirow[b]{2}{*}{ Group } & \multicolumn{3}{|c|}{ CNR1 rs2023239 } & & & \multirow{2}{*}{$\begin{array}{l}\text { Comparison }^{a} \\
\text { CC+CT vs TT }\end{array}$} & \multirow{2}{*}{$\begin{array}{l}\text { OR }^{b} \\
1.903\end{array}$} & \multicolumn{2}{|c|}{$95 \% \mathrm{Cl}$} & \multirow{3}{*}{$\begin{array}{c}\mathbf{p}^{\mathbf{c}} \\
\mathbf{0 . 0 0 1} \\
0.920\end{array}$} \\
\hline & \multicolumn{3}{|c|}{ Genotype } & \multicolumn{2}{|c|}{ Allele } & & & 1.290 & 2.808 & \\
\hline & $\pi$ & CT & $\mathrm{CC}$ & $\mathrm{T}$ & $\mathrm{C}$ & $\mathrm{CC}$ vs $\mathrm{CT}+\mathrm{TT}$ & 1.066 & 0.305 & 3.726 & \\
\hline Controls n & 222 & 52 & 5 & 496 & 62 & CC vs Tा & 1.261 & 0.360 & 4.426 & 0.716 \\
\hline$\%$ & 79.6 & 18.6 & 1.8 & 88.9 & 11.1 & C vs T & 1.681 & 1.188 & 2.380 & 0.003 \\
\hline GTS n & 176 & 81 & 5 & 433 & 91 & CT vs TT & 1.965 & 1.316 & 2.933 & 0.001 \\
\hline \multirow[t]{2}{*}{$\%$} & 67.2 & 30.9 & 1.9 & 82.6 & 17.4 & CC vs CT & 0.642 & 0.177 & 2.327 & 0.497 \\
\hline & \multicolumn{3}{|c|}{ CNR1 rs2180619 } & & & Comparison $^{a}$ & $\mathrm{OR}^{\mathrm{b}}$ & \multicolumn{2}{|c|}{$95 \% \mathrm{Cl}$} & $p^{c}$ \\
\hline \multirow[t]{2}{*}{ Group } & \multicolumn{3}{|c|}{ Genotype } & \multicolumn{2}{|c|}{ Allele } & $G G+A G$ vs $A A$ & 1.281 & 0.903 & 1.818 & 0.164 \\
\hline & $A A$ & $A G$ & $G G$ & $A$ & $G$ & $G G$ vs $A G+A A$ & 0.866 & 0.531 & 1.412 & 0.563 \\
\hline Controls n & 112 & 126 & 41 & 350 & 208 & GG vs $A A$ & 1.032 & 0.606 & 1.758 & 0.909 \\
\hline$\%$ & 40.1 & 45.2 & 14.7 & 62.7 & 37.3 & G vs A & 1.090 & 0.853 & 1.393 & 0.491 \\
\hline GTS n & 90 & 138 & 34 & 318 & 206 & $A G$ vs $A A$ & 1.363 & 0.944 & 1.969 & 0.099 \\
\hline \multirow[t]{2}{*}{$\%$} & 34.4 & 52.7 & 13.0 & 60.7 & 39.3 & GG vs $A G$ & 0.757 & 0.453 & 1.267 & 0.289 \\
\hline & \multicolumn{3}{|c|}{ CNR1 rs806379 } & & & Comparison $^{a}$ & $\mathrm{OR}^{\mathrm{b}}$ & \multicolumn{2}{|c|}{$95 \% \mathrm{Cl}$} & $p^{c}$ \\
\hline \multirow[t]{2}{*}{ Group } & \multicolumn{3}{|c|}{ Genotype } & \multicolumn{2}{|c|}{ Allele } & TT+AT vs AA & 0.837 & 0.579 & 1.210 & 0.344 \\
\hline & $\mathrm{AA}$ & AT & $\pi$ & $A$ & $\mathrm{~T}$ & $\Pi \mathrm{v} v \mathrm{AT}+\mathrm{AA}$ & 1.204 & 0.807 & 1.797 & 0.362 \\
\hline Controls n & 78 & 141 & 60 & 297 & 261 & Tा vs AA & 1.018 & 0.638 & 1.625 & 0.940 \\
\hline$\%$ & 28.0 & 50.5 & 21.5 & 53.2 & 46.8 & T vs A & 0.992 & 0.781 & 1.259 & 0.945 \\
\hline GTS & 83 & 114 & 65 & 280 & 244 & AT vs AA & 0.760 & 0.512 & 1.129 & 0.173 \\
\hline \multicolumn{11}{|l|}{$\mathrm{n}$} \\
\hline \multirow[t]{2}{*}{$\%$} & 31.7 & 43.5 & 24.8 & 53.4 & 46.6 & Tा vs AT & 1.340 & 0.873 & 2.058 & 0.181 \\
\hline & \multicolumn{3}{|c|}{ CNR1 rs1049353 } & & & Comparison $^{a}$ & $\mathrm{OR}^{\mathrm{b}}$ & \multicolumn{2}{|c|}{$95 \% \mathrm{Cl}$} & $p^{c}$ \\
\hline \multirow[t]{2}{*}{ Group } & \multicolumn{3}{|c|}{ Genotype } & \multicolumn{2}{|c|}{ Allele } & $T+C T$ vs $C C$ & 0.876 & 0.622 & 1.232 & 0.445 \\
\hline & $\mathrm{CC}$ & CT & $\Pi$ & C & $\mathrm{T}$ & $\Pi$ vs $\mathrm{CT}+\mathrm{CC}$ & 0.626 & 0.269 & 1.456 & 0.273 \\
\hline Controls n & 156 & 108 & 15 & 420 & 138 & Tा vs CC & 0.604 & 0.257 & 1.421 & 0.244 \\
\hline$\%$ & 55.9 & 38.7 & 5.4 & 75.1 & 24.9 & T vs C & 0.865 & 0.653 & 1.147 & 0.314 \\
\hline \multicolumn{11}{|l|}{ GTS } \\
\hline $\mathrm{n}$ & 155 & 98 & 9 & 408 & 116 & CT vs CC & 0.913 & 0.642 & 1.299 & 0.613 \\
\hline$\%$ & 59.2 & 37.4 & 3.4 & 78.0 & 22.0 & TT vs CT & 0.661 & 0.277 & 1.579 & 0.349 \\
\hline
\end{tabular}

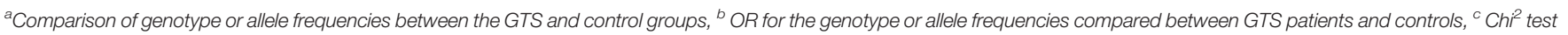


TABLE 4 | Genotypic and allelic association analysis of studied CNR1 variants with most common co-morbid disorders in patients with Gilles de la Tourette syndrome.

\begin{tabular}{|c|c|c|c|c|c|c|c|c|c|c|}
\hline \multirow{3}{*}{$\begin{array}{l}\text { Co-morbid disorder } \\
A D H D\end{array}$} & \multicolumn{5}{|c|}{ CNR1 rs2023239 } & \multirow{3}{*}{$\begin{array}{l}\text { Comparison }^{\text {a }} \\
\text { CC+CT vs TT } \\
\text { CC vs CT+TT }\end{array}$} & \multirow{3}{*}{$\begin{array}{l}\text { OR }^{\mathbf{b}} \\
1.289 \\
0.469\end{array}$} & \multicolumn{2}{|c|}{$95 \% \mathrm{Cl}$} & \multirow{3}{*}{$\frac{p^{\mathbf{c}}}{0.354}$} \\
\hline & \multicolumn{3}{|c|}{ Genotype } & \multicolumn{2}{|c|}{ Allele } & & & 0.753 & 2.206 & \\
\hline & $\pi$ & CT & $\mathrm{CC}$ & $\mathrm{T}$ & C & & & 0.052 & 4.261 & \\
\hline Absent, n & 118 & 49 & 4 & 285 & 57 & CC vs Tा & 0.518 & 0.057 & 4.737 & 0.553 \\
\hline$\%$ & 69.0 & 28.7 & 2.3 & 83.3 & 16.7 & C vs T & 1.164 & 0.728 & 1.862 & 0.525 \\
\hline Present, n & 57 & 32 & 1 & 146 & 34 & CT vs TT & 1.352 & 0.783 & 2.335 & 0.279 \\
\hline$\%$ & 63.3 & 35.6 & 1.1 & 81.1 & 18.9 & CC vs CT & 0.383 & 0.041 & 3.582 & 0.384 \\
\hline \multirow[t]{2}{*}{ OCD/OCB } & \multicolumn{3}{|c|}{ Genotype } & \multicolumn{2}{|c|}{ Allele } & $\mathrm{CC}+\mathrm{CT}$ vs TT & 0.619 & 0.367 & 1.044 & 0.071 \\
\hline & $\pi$ & CT & $\mathrm{CC}$ & $\mathrm{T}$ & C & $\mathrm{CC}$ vs $\mathrm{CT}+\mathrm{TT}$ & 1.597 & 0.262 & 9.717 & 0.608 \\
\hline Absent, n & 83 & 49 & 2 & 215 & 53 & CC vs TT & 1.353 & 0.221 & 8.299 & 0.743 \\
\hline$\%$ & 61.9 & 36.6 & 1.5 & 80.2 & 19.8 & $\mathrm{C}$ vs T & 0.714 & 0.452 & 1.128 & 0.147 \\
\hline Present, n & 92 & 32 & 3 & 216 & 38 & CT vs TT & 0.589 & 0.345 & 1.006 & 0.052 \\
\hline$\%$ & 72.4 & 25.2 & 2.4 & 85.0 & 15.0 & CC vs CT & 2.297 & 0.363 & 14.518 & 0.365 \\
\hline \multirow[t]{2}{*}{ Anxiety } & & enotyp & & & & $\mathrm{CC}+\mathrm{CT}$ vs TT & 0.947 & 0.544 & 1.649 & 0.848 \\
\hline & $\pi$ & CT & $\mathrm{CC}$ & $\mathrm{T}$ & C & $\mathrm{CC}$ vs $\mathrm{CT}+\mathrm{TT}$ & 3.241 & 0.531 & 19.772 & 0.179 \\
\hline Absent, n & 118 & 57 & 2 & 293 & 61 & CC vs TT & 3.105 & 0.505 & 19.106 & 0.200 \\
\hline$\%$ & 66.7 & 32.2 & 1.1 & 82.8 & 17.2 & $\mathrm{C}$ vs T & 1.044 & 0.645 & 1.690 & 0.860 \\
\hline Present, n & 57 & 24 & 3 & 138 & 30 & CT vs TT & 0.872 & 0.492 & 1.545 & 0.638 \\
\hline$\%$ & 67.9 & 28.6 & 3.6 & 82.1 & 17.9 & CC vs CT & 3.563 & 0.559 & 22.695 & 0.156 \\
\hline Depression & & enotyp & & & & $\mathrm{CC}+\mathrm{CT}$ vs TT & 0.841 & 0.394 & 1.793 & 0.653 \\
\hline & $\pi$ & CT & $\mathrm{CC}$ & $\mathrm{T}$ & C & $\mathrm{CC}$ vs $\mathrm{CT}+\mathrm{TT}$ & 1.528 & 0.166 & 14.059 & 0.706 \\
\hline Absent, n & 149 & 71 & 4 & 369 & 79 & CC vs TT & 1.433 & 0.154 & 13.331 & 0.751 \\
\hline$\%$ & 66.5 & 31.7 & 1.8 & 82.4 & 17.6 & C vs T & 0.904 & 0.465 & 1.756 & 0.766 \\
\hline Present, n & 26 & 10 & 1 & 62 & 12 & CT vs TT & 0.807 & 0.369 & 1.765 & 0.591 \\
\hline$\%$ & 70.3 & 27.0 & 2.7 & 83.8 & 16.2 & CC vs CT & 1.775 & 0.180 & 17.513 & 0.619 \\
\hline ADHD & & enotyp & & & & $G G+A G$ vs $A A$ & 0.864 & 0.506 & 1.473 & 0.590 \\
\hline & $\mathrm{AA}$ & $A G$ & GG & $A$ & $\mathrm{G}$ & $G G$ vs $A G+A A$ & 0.544 & 0.235 & 1.257 & 0.150 \\
\hline Absent, n & 57 & 88 & 26 & 202 & 140 & GG vs $A A$ & 0.531 & 0.216 & 1.308 & 0.165 \\
\hline$\%$ & 33.3 & 51.5 & 15.2 & 59.1 & 40.9 & G vs $A$ & 0.816 & 0.562 & 1.184 & 0.283 \\
\hline Present, n & 33 & 49 & 8 & 115 & 65 & $A G$ vs $A A$ & 0.962 & 0.553 & 1.672 & 0.890 \\
\hline$\%$ & 36.7 & 54.4 & 8.9 & 63.9 & 36.1 & GG vs AG & 0.553 & 0.232 & 1.314 & 0.175 \\
\hline OCD/OCB & & enotyp & & & & $G G+A G$ vs $A A$ & 0.986 & 0.592 & 1.643 & 0.957 \\
\hline & $\mathrm{AA}$ & $A G$ & GG & $A$ & $\mathrm{G}$ & $G G$ vs $A G+A A$ & 0.531 & 0.251 & 1.125 & 0.095 \\
\hline Absent, n & 46 & 66 & 22 & 158 & 110 & GG vs AA & 0.570 & 0.252 & 1.289 & 0.175 \\
\hline$\%$ & 34.3 & 49.3 & 16.4 & 59.0 & 41.0 & G vs $A$ & 0.858 & 0.604 & 1.220 & 0.394 \\
\hline Present, $\mathrm{n}$ & 44 & 71 & 12 & 159 & 95 & $A G$ vs $A A$ & 1.125 & 0.661 & 1.915 & 0.665 \\
\hline$\%$ & 34.7 & 55.9 & 9.5 & 62.6 & 37.4 & GG vs $A G$ & 0.507 & 0.233 & 1.105 & 0.084 \\
\hline Anxiety & & enotyp & & & & $G G+A G$ vs $A A$ & 0.855 & 0.497 & 1.470 & 0.571 \\
\hline & $A A$ & $A G$ & GG & $A$ & $\mathrm{G}$ & $G G$ vs $A G+A A$ & 0.730 & 0.324 & 1.641 & 0.444 \\
\hline Absent, $\mathrm{n}$ & 59 & 93 & 25 & 211 & 143 & GG vs AA & 0.685 & 0.285 & 1.647 & 0.397 \\
\hline$\%$ & 33.3 & 52.5 & 14.1 & $59 . \%$ & 40.4 & G vs A & 0.863 & 0.591 & 1.260 & 0.445 \\
\hline Present, $\mathrm{n}$ & 31 & 44 & 9 & 106 & 62 & $A G$ vs $A A$ & 0.900 & 0.512 & 1.582 & 0.715 \\
\hline$\%$ & 36.9 & 52.4 & 10.7 & $63 . \%$ & 36.9 & GG vs AG & 0.761 & 0.328 & 1.766 & 0.524 \\
\hline Depression & & enotyp & & & & $G G+A G$ vs $A A$ & 0.844 & 0.411 & 1.733 & 0.643 \\
\hline & $\mathrm{AA}$ & $A G$ & GG & $A$ & $\mathrm{G}$ & $G G$ vs $A G+A A$ & 0.784 & 0.259 & 2.370 & 0.666 \\
\hline Absent, $\mathrm{n}$ & 76 & 118 & 30 & 270 & 178 & GG vs AA & 0.724 & 0.220 & 2.376 & 0.593 \\
\hline$\%$ & 33.9 & 52.7 & 13.4 & 60.3 & 39.7 & G vs A & 0.871 & 0.523 & 1.451 & 0.596 \\
\hline Present, $\mathrm{n}$ & 14 & 19 & 4 & 47 & 27 & $A G$ vs $A A$ & 0.874 & 0.414 & 1.847 & 0.724 \\
\hline$\%$ & 37.8 & 51.4 & 10.8 & 63.5 & 36.5 & GG vs $A G$ & 0.828 & 0.262 & 2.616 & 0.748 \\
\hline Depression & & enotyp & & & & TT+CT vs CC & 0.997 & 0.491 & 2.024 & 0.992 \\
\hline & $\mathrm{CC}$ & CT & $\pi$ & $\mathrm{C}$ & $\mathrm{T}$ & $\pi$ vs $\mathrm{CT}+\mathrm{CC}$ & 0.000 & - & - & 0.243 \\
\hline Absent, n & 133 & 83 & 8 & 349 & 99 & Tा vs CC & 0.000 & - & - & 0.252 \\
\hline$\%$ & 59.4 & 37.1 & 3.6 & 77.9 & 22.1 & T vs C & 0.896 & 0.487 & 1.648 & 0.724 \\
\hline Present, $n$ & 22 & 15 & 0 & 59 & 15 & CT vs CC & 1.093 & 0.536 & 2.225 & 0.807 \\
\hline$\%$ & 59.5 & 40.5 & 0.0 & 79.7 & 20.3 & TT vs CT & 0.000 & - & - & 0.232 \\
\hline Anxiety & & enotyp & & & & $\pi+\mathrm{CT}$ vs $\mathrm{CC}$ & 1.232 & 0.728 & 2.085 & 0.436 \\
\hline & $\mathrm{CC}$ & $\mathrm{CT}$ & $\pi$ & C & $\mathrm{T}$ & $\Pi$ vs $\mathrm{CT}+\mathrm{CC}$ & 1.274 & 0.297 & 5.462 & 0.744 \\
\hline Absent, n & 108 & 64 & 5 & 280 & 74 & Tा vs CC & 1.379 & 0.316 & 6.007 & 0.668 \\
\hline$\%$ & 61.0 & 36.2 & 2.8 & 79.1 & 20.9 & T vs C & 1.182 & 0.763 & 1.832 & 0.453 \\
\hline Present, $\mathrm{n}$ & 47 & 34 & 3 & 128 & 40 & CT vs CC & 1.221 & 0.712 & 2.092 & 0.468 \\
\hline$\%$ & 56.0 & 40.5 & 3.6 & 76.2 & 23.8 & TT vs CT & 1.129 & 0.254 & 5.014 & 0.873 \\
\hline OCD/OCB & & enotyp & & & & TT+CT vs CC & 0.796 & 0.485 & 1.307 & 0.367 \\
\hline & $\mathrm{CC}$ & CT & $\pi$ & C & $\mathrm{T}$ & Tा vs $\mathrm{CT}+\mathrm{CC}$ & 0.624 & 0.146 & 2.667 & 0.521 \\
\hline Absent, n & 76 & 53 & 5 & 205 & 63 & Tा vs CC & 0.577 & 0.133 & 2.499 & 0.458 \\
\hline
\end{tabular}


TABLE 4 | Continued

\begin{tabular}{|c|c|c|c|c|c|c|c|c|c|c|}
\hline \multirow{2}{*}{$\begin{array}{l}\text { Co-morbid disorder } \\
\%\end{array}$} & \multicolumn{5}{|c|}{ CNR1 rs2023239 } & \multirow{2}{*}{$\begin{array}{l}\text { Comparison }^{\mathbf{a}} \\
\text { T vs C }\end{array}$} & \multirow{2}{*}{\begin{tabular}{|l} 
OR $^{\mathbf{b}}$ \\
0.817
\end{tabular}} & \multicolumn{2}{|c|}{$95 \% \mathrm{Cl}$} & \multirow{2}{*}{$\frac{p^{\mathbf{c}}}{0.343}$} \\
\hline & 56.7 & 39.6 & 3.7 & 76.5 & 23.5 & & & 0.539 & 1.241 & \\
\hline Present, $\mathrm{n}$ & 79 & 45 & 3 & 203 & 51 & CT vs CC & 0.817 & 0.492 & 1.356 & 0.434 \\
\hline$\%$ & 62.2 & 35.4 & 2.4 & 79.9 & 20.1 & Tा vs CT & 0.707 & 0.160 & 3.121 & 0.646 \\
\hline \multirow[t]{2}{*}{ ADHD } & \multicolumn{3}{|c|}{ Genotype } & \multicolumn{2}{|c|}{ Allele } & $T+\mathrm{CT}$ vs $\mathrm{CC}$ & 1.107 & 0.659 & 1.859 & 0.701 \\
\hline & $\mathrm{CC}$ & CT & $\pi$ & $\mathrm{C}$ & $\mathrm{T}$ & TT vs CT+CC & 1.942 & 0.474 & 7.955 & 0.348 \\
\hline Absent, $\mathrm{n}$ & 103 & 64 & 4 & 270 & 72 & Tा vs CC & 1.981 & 0.476 & 8.239 & 0.339 \\
\hline$\%$ & 60.2 & 37.4 & 2.3 & 79.0 & 21.1 & T vs C & 1.141 & 0.741 & 1.759 & 0.549 \\
\hline Present, $\mathrm{n}$ & 52 & 34 & 4 & 138 & 42 & CT vs CC & 1.052 & 0.617 & 1.793 & 0.851 \\
\hline$\%$ & 57.8 & 37.8 & 4.4 & 76.7 & 23.3 & TT vs CT & 1.882 & 0.443 & 8.000 & 0.385 \\
\hline \multirow[t]{2}{*}{ Anxiety } & \multicolumn{3}{|c|}{ Genotype } & \multicolumn{2}{|c|}{ Allele } & TT+AT vs $A A$ & 1.445 & 0.811 & 2.575 & 0.210 \\
\hline & $\mathrm{AA}$ & AT & $\pi$ & $A$ & $\mathrm{~T}$ & $\Pi$ vs $A T+A A$ & 1.106 & 0.610 & 2.006 & 0.741 \\
\hline Absent, $\mathrm{n}$ & 60 & 74 & 43 & 194 & 160 & TT vs AA & 1.395 & 0.687 & 2.835 & 0.356 \\
\hline$\%$ & 33.9 & 41.8 & 24.3 & 54.8 & 45.2 & T vs A & 1.213 & 0.839 & 1.752 & 0.304 \\
\hline Present, n & 22 & 40 & 22 & 84 & 84 & AT vs AA & 1.474 & 0.792 & 2.745 & 0.220 \\
\hline$\%$ & 26.2 & 47.6 & 26.2 & 50.0 & 50.0 & TT vs AT & 0.947 & 0.498 & 1.798 & 0.867 \\
\hline \multirow[t]{2}{*}{ Depression } & \multicolumn{3}{|c|}{ Genotype } & \multicolumn{2}{|c|}{ Allele } & TT+AT vs $A A$ & 0.716 & 0.348 & 1.475 & 0.364 \\
\hline & $\mathrm{AA}$ & AT & $\pi$ & A & $\mathrm{T}$ & $\Pi$ vs $A T+A A$ & 0.668 & 0.278 & 1.603 & 0.363 \\
\hline Absent, $\mathrm{n}$ & 68 & 98 & 68 & 234 & 214 & Tा vs AA & 0.586 & 0.222 & 1.550 & 0.278 \\
\hline$\%$ & 30.4 & 43.8 & 30.4 & 52.2 & 47.8 & T vs A & 0.746 & 0.452 & 1.229 & 0.248 \\
\hline Present, n & 14 & 16 & 14 & 44 & 30 & AT vs AA & 0.793 & 0.363 & 1.732 & 0.560 \\
\hline$\%$ & 37.8 & 43.2 & 37.8 & 59.5 & 40.5 & TT vs AT & 0.739 & 0.287 & 1.903 & 0.530 \\
\hline \multirow[t]{2}{*}{ OCD/OCB } & \multicolumn{3}{|c|}{ Genotype } & \multicolumn{2}{|c|}{ Allele } & $\pi+A T$ vs $A A$ & 0.925 & 0.548 & 1.560 & 0.769 \\
\hline & $\mathrm{AA}$ & AT & $\pi$ & $A$ & $\mathrm{~T}$ & $\Pi$ vs $A T+A A$ & 1.031 & 0.588 & 1.807 & 0.915 \\
\hline Absent, $\mathrm{n}$ & 41 & 60 & 33 & 142 & 126 & TT vs AA & 0.970 & 0.506 & 1.859 & 0.926 \\
\hline$\%$ & 30.6 & 44.8 & 24.6 & 53.0 & 47.0 & T vs A & 0.978 & 0.693 & 1.379 & 0.898 \\
\hline Present, n & 41 & 54 & 32 & 136 & 118 & AT vs AA & 0.900 & 0.510 & 1.588 & 0.716 \\
\hline$\%$ & 32.3 & 42.5 & 25.2 & 53.5 & 46.5 & TT vs AT & 1.077 & 0.586 & 1.982 & 0.810 \\
\hline \multirow[t]{2}{*}{ ADHD } & \multicolumn{3}{|c|}{ Genotype } & \multicolumn{2}{|c|}{ Allele } & TT+AT vs AA & 1.822 & 1.020 & 3.255 & 0.041 \\
\hline & $\mathrm{AA}$ & AT & $\Pi$ & $A$ & $\mathrm{~T}$ & Tा vs $A T+A A$ & 1.375 & 0.771 & 2.454 & 0.280 \\
\hline Absent, $\mathrm{n}$ & 61 & 71 & 39 & 193 & 149 & Tा vs AA & 1.937 & 0.960 & 3.906 & 0.063 \\
\hline$\%$ & 35.7 & 41.5 & 22.8 & 56.4 & 43.6 & T vs A & 1.448 & 1.008 & 2.080 & 0.045 \\
\hline Present, n & 21 & 43 & 26 & 85 & 95 & AT vs AA & 1.759 & 0.943 & 3.283 & 0.075 \\
\hline$\%$ & 23.3 & 47.8 & 28.9 & 47.2 & 52.8 & TT vs AT & 1.101 & 0.590 & 2.055 & 0.763 \\
\hline
\end{tabular}

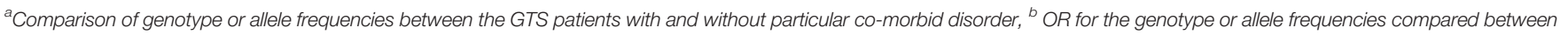
GTS patients with and without particular co-morbid disorder, ${ }^{\circ}$ Chi ${ }^{2}$ test.

show differences with healthy controls. Our study is the first to examine common SNP variants of CNR1 gene in a group of GTS patients. A significant association was found both in the allelic and genotypic analysis between the risk of GTS and the rs2023239 polymorphism. Multivariate analysis proved that $\mathrm{CC}+\mathrm{CT}$ genotypes are gender-independent factors associated with a higher GTS risk. Our study shows that GTS is associated with rs2023239 polymorphism along with various others neuropsychiatric disorders, such as the cannabis dependence (Benzinou et al., 2008), eating disorders (Yu et al., 2013), schizophrenia (Wiskerke et al., 2012), impulsivity (Juhasz et al., 2009), depression (Ruiz-Contreras et al., 2017), and migraine (Ketcherside et al., 2017). We can only speculate on the molecular mechanism underlying the role of this variant in the pathogenesis of GTS. rs2023239 allele C was found to be associated with increased CB1 receptor density (Janik et al., 2015) which could modulate neurotransmission. It is unlikely that this intronic variant directly affects the splicing, as it is too distant the adjacent exon (122bp). Human Splicing Finder predicts that the minor allele $\mathrm{C}$ does not create any new potential splice sites or new potential branch points compared to the common $\mathrm{T}$ allele. Additionally, this variant could affect gene expression either directly or through a linkage to functional variant(s) located in regulatory regions.
Our earlier association studies in Polish patients with GTS have revealed a relationship between some SNPs of BTBD9, ADORA1 and ADORA2A genes and co-morbid psychiatric disorders (Muller-Vahl et al., 1999; Martiny, 2017). Therefore, we hypothesized that $C N R 1$ variants could also contribute to the disease complexity predisposing the patients to psychiatric comorbidities as all the CNR1 polymorphisms studied here had been found to be associated with various psychiatric disorders (Table 1). However, both genotypic and allelic association analyses of all the CNR1variants examined, including rs2023239 associated with a higher risk of GTS, failed to demonstrate a significant association with any psychiatric comorbidity. The only statistically significant association found, that of co-morbid ADHD with rs806379, should probably be regarded as a false positive because of its marginal significance $(0.05>\mathrm{p}>0.04)$ in multiple statistical tests performed (4 SNPs x 4 disorders x 6 models).

A number of open as well as randomized controlled studies and case reports support the premise that the use of CBM improves not only tics, but also ADHD (Abi-Jaoude et al., 2017), OCB/OCD (Hasan et al., 2010; Denys et al., 2013), depression (Yoon et al., 2007) and anxiety (Jakubovski and Müller-Vahl, 2017). Our study does not confirm that this beneficial effect of CMB on co-morbid disorders in GTS could 
be related to CNR1 SNPs as we did not find any association of the CNR1 variants with co-morbidity. Additionally, we did not find any correlation of CNR1 variants with any clinical data, such as age of tic onset, tic severity measured with YGTSS or familial history of tics and OCD/OCB. It can therefore be speculated that while rs2023239 contributes to the pathogenesis of tics per se, other genetic and environmental factors contribute to further clinical features of GTS.

The present results on the CNR1 variants could also be interpreted in relation to an earlier SNP study by our group, in which we found association of ADORA1 and ADORA2A variants with a higher risk of GTS (Muller-Vahl et al., 1999). In this context we note that abnormality of the dopaminergic system has been confirmed to be one of the primary causes of GTS (Singer et al., 1982; Sandyk and Bamford, 1987; Ferré et al., 2009; Ferré et al., 2010; Filbey et al., 2010), both adenosine and endocannabinoids act as modulators of dopamine neurotransmission in the striatum (Müller et al., 2007) and it has been suggested that $\mathrm{CB} 1$ receptors form heteromers with dopamine D2 and adenosine A2A receptors (Ehlers et al., 2007). Although we do not know the functional significance of the minor variant of rs2023239 in GTS, we put forward the hypothesis that it could be related to faulty cannabinoid transmission due to lower expression of the CB1 receptor or reduced receptor's affinity for the endocannabinoid ligand. This, in turn, could lead to reduction of adenosinergic signaling and over-activity of dopaminergic transmission, and finally aggravate tics. Another possible mechanism is a direct enhancement of dopaminergic transmission as a consequence of limited endocannabinoid activity, without an involvement of adenosine signaling.

In conclusion, the obtained results indicate that the $\mathrm{C}$ allele of the rs2023239 polymorphism of CNR1 gene is a risk factor of GTS in the Polish population, associated with the occurrence of tics, but not with the co-existing psychiatric symptoms. The results also support the hypothesis of an ECS involvement in the pathogenesis of GTS. Nevertheless, our finding is preliminary and needs to be replicated in an independent cohort. Further research is needed to determine functional significance of the variant.

\section{LIMITATIONS}

The following limitations of the study have to be addressed: 1) the analyzed variants can be in linkage disequilibrium with true risk conferring variants located outside analyzed regions; 2) the study group was relatively small; 3) the patients were evaluated for co-morbid disorders only once (one-time registration) and it cannot be excluded that psychiatric disorders could develop over time and additionally it was impossible to measure the YGTSS in all patients; there is a possibility of false negative results in rs2180619, rs806379, and rs1049353 CNR1 polymorphisms; 4) some clinical data, especially on adult patients, were subject to a recall bias, ; 5) we have not included the comparison with the variants previously associated with GTS and this should be taken into consideration in the future studies; and 6) our finding needs to be replicated using study groups from different populations.

\section{DATA AVAILABILITY STATEMENT}

The raw data supporting the conclusions of this article will be made available by the authors, without undue reservation, to any qualified researcher. These are also included in Supplementary Material.

\section{ETHICS STATEMENT}

The study has been approved by the Ethics Committee of the Medical University of Warsaw (KB/2/2007, KB/53/A/2010, KB/ $63 / A / 2018)$ and has therefore been performed in accordance with the ethical standards laid down in the 1964 Declaration of Helsinki and its later amendments. All participants aged $\geq 18$ years signed an informed consent form prior to their inclusion in the study, and legal guardians gave a written consent on behalf of individuals under 18 .

\section{AUTHOR CONTRIBUTIONS}

Research project: Conception (PJ), Organization (PJ, CŻ), Execution (JF), Creation of electronic database (TD, NS, PJ), Clinical examination: (PJ), Manuscript Preparation: Writing of the first draft (NS, PJ, JF, KS), Review and Critique (CŻ, PJ, KS, JF, NS, TD).

\section{FUNDING}

This study was kindly supported by a grant provided by Medical University of Warsaw (1WC/NM1/17) and by a grant funded by National Science Center (UMO-2016/23/B/NZ2/03030).

\section{ACKNOWLEDGMENTS}

We thank all patients for participation in this study.

\section{SUPPLEMENTARY MATERIAL}

The Supplementary Material for this article can be found online at: https://www.frontiersin.org/articles/10.3389/fgene.2020. 00125/full\#supplementary-material

SUPPLEMENTARY TABLE 1 | The raw data used for analysis in this study. 


\section{REFERENCES}

Abi-Jaoude, E., Chen, L., Cheung, P., Bhikram, T., and Sandor, P. (2017). Preliminary evidence on cannabis effectiveness and tolerability for adults with tourette syndrome. J. Neuropsychiatry Clin. Neurosci. 29, 391-400. doi: 10.1176/appi.neuropsych.16110310

Benzinou, M., Chèvre, J. C., Ward, K. J., Lecoeur, C., Dina, C., Lobbens, S., et al. (2008). Endocannabinoid receptor 1 gene variations increase risk for obesity and modulate body mass index in European populations. Hum. Mol. Genet. 17 (13), 1916-1921. doi: 10.1093/hmg/ddn089

Berding, G., Müller-Vahl, K., Schneider, U., Gielow, P., Fitschen, J., Stuhrmann, M., et al. (2004). [123I]AM281 single-photon emission computed tomography imaging of central cannabinoid CB1receptors before and after $\Delta 9$ tetrahydrocannabinol therapy and whole-body scanning for assessment of radiation dose in Tourette patients. Biol. Psychiatry 55 (9), 904-915. doi: 10.1016/j.biopsych.2004.01.005

Brunnauer, A., Segmiller, F. M., Volkamer, T., Laux, G., Müller, N., and Dehning, S. (2011). Cannabinoids improve driving ability in a Tourette's patient. Psychiatry Res. 190, 382. doi: 10.1016/j.psychres.2011.05.033

Buchmann, A. F., Hohm, E., Witt, S. H., Blomeyer, D., Jennen-Steinmetz, C., Schmidt, M. H., et al. (2015). Role of CNR1 polymorphisms in moderating the effects of psychosocial adversity on impulsivity in adolescents. J. Neural Transm. 122 (3), 455-463. doi: 10.1007/s00702-014-1266-3

Chen, X., Williamson, V. S., An, S. S., Hettema, J. M., Aggen, S. H., Neale, M. C., et al. (2008). Cannabinoid receptor 1 gene association with nicotine dependence. Arch. Gen. Psychiatry 65 (7), 816-824. doi: 10.1001/archpsyc. 65.7 .816

Denys, D., de Vries, F., Cath, D., Figee, M., Vulink, N., Veltman, D. J., et al. (2013). Dopaminergic activity in Tourette syndrome and obsessive-compulsive disorder. Eur. Neuropsychopharmacol. [Internet] 23 (11), 1423-1431. doi: 10.1016/j.euroneuro.2013.05.012

Domschke, K., Dannlowski, U., Ohrmann, P., Lawford, B., Bauer, J., Kugel, H., et al. (2008). Cannabinoid receptor 1 (CNR1) gene: impact on antidepressant treatment response and emotion processing in major depression. Eur. Neuropsychopharmacol. 18 (10), 751-759. doi: 10.1016/j.euroneuro. 2008.05.003

Ehlers, C. L., Slutske, W. S., Lind, P. A., and Wilhelmsen, K. C. (2007). Association between single nucleotide polymorphisms in the cannabinoid receptor gene (CNR1) and impulsivity in Southwest California Indians. Twin Res. Hum. Genet. 10 (6), 805-811. doi: 10.1375/twin.10.6.805

Evans, D. E., Sutton, S. K., Jentink, K. G., Lin, H. Y., Park, J. Y., and Drobes, D. J. (2016). Cannabinoid receptor 1 (CNR1) gene variant moderates neural index of cognitive disruption during nicotine withdrawal. Genes Brain Behav. 15 (7), 621-626. doi: 10.1111/gbb.12311

Ferré, S., Goldberg, S. R., Lluis, C., and Franco, R. (2009). Looking for the role of cannabinoid receptor heteromers in striatal function. Neuropharmacology 56, 226-234. doi: 10.1016/j.neuropharm.2008.06.076

Ferré, S., Lluís, C., Justinova, Z., Quiroz, C., Orru, M., Navarro, G., et al. (2010). Adenosine-cannabinoid receptor interactions. implications for striatal function. Br. J. Pharmacol. 160, 443-453. doi: 10.1111/j.14765381.2010.00723.x

Filbey, F. M., Schacht, J. P., Myers, U. S., Chavez, R. S., and Hutchison, K. E. (2010). Individual and additive effects of the CNR1 and FAAH genes on brain response to marijuana cues. Neuropsychopharmacology 35 (4), 967-975. doi: 10.1038/npp.2009.200

Gadzicki, D., Müller-Vahl, K., and Stuhrmann, M. (1999). A frequent polymorphism in the coding exon of the human cannabinoid receptor (CNR1) gene. Mol. Cell Probes. 13 (4), 321-323. doi: 10.1006/mcpr.1999.0249

Gadzicki, D., Müller-Vahl, K. R., Heller, D., Ossege, S., Nöthen, M. M., Hebebrand, J., et al. (2004). Tourette syndrome is not caused by mutations in the central cannabinoid receptor (CNR1) gene. Am. J. Med. Genet. B Neuropsychiatr. Genet. [Internet]. 127B (1), 97-103. doi: 10.1002/ajmg.b.20159

Haber, S. N., and Wolfer, D. (1992). Basal ganglia peptidergic staining in Tourette syndrome. A follow-up study. Adv. Neurol. 58, 145-150.

Hasan, A., Rothenberger, A., Münchau, A., Wobrock, T., Falkai, P., and Roessner, V. (2010). Oral ??9-tetrahydrocannabinol improved refractory Gilles de la Tourette syndrome in an adolescent by increasing intracortical inhibition: a case report. J. Clin. Psychopharmacol. 30 (2), 190-192. doi: 10.1097/JCP.0b013e3181d236ec

Haughey, H. M., Marshall, E., Schacht, J. P., Louis, A., and Hutchison, K. E. (2008). Marijuana withdrawal and craving: Influence of the cannabinoid receptor 1 (CNR1) and fatty acid amide hydrolase (FAAH) genes. Addiction 103 (10), 1678-1686. doi: 10.1111/j.1360-0443.2008.02292.x

Hemming, M., and Yellowlees, P. M. (1993). Effective treatment of Tourette's syndrome with marijuana. J. Psychopharmacol. 7 (4), 389-391. doi: 10.1177/ 026988119300700411

Hindocha, C., Freeman, T. P., Schafer, G., Gardner, C., Bloomfield, M. A. P., Bramon, E., et al. (2019). Acute effects of cannabinoids on addiction endophenotypes are moderated by genes encoding the CB1 receptor and FAAH enzyme. Addict. Biol. 1-11. doi: 10.1111/adb.12762

Icick, R., Peoc', H. K., Karsinti, E., Ksouda, K., Hajj, A., Bloch, V., et al. (2015). A cannabinoid receptor 1 polymorphism is protective against major depressive disorder in methadone-maintained outpatients. Am. J. Addict. 24 (7), 613-620. doi: $10.1111 /$ ajad.12273

Jakubovski, E., and Müller-Vahl, K. (2017). Speechlessness in Gilles de la Tourette syndrome: Cannabis-based medicines improve severe vocal blocking tics in two patients. Int. J. Mol. Sci. 18 (8), 1-6. doi: 10.3390/ijms18081739

Janik, P., Berdyński, M., Safranow, K., and Zekanowski, C. (2014). The BTBD9 gene polymorphisms in Polish patients with Gilles de la Tourette syndrome. Acta Neurobiol. Exp. (Wars). 74 (2), 218-226.

Janik, P., Berdyński, M., Safranow, K., Zekanowski, C., and Blum, D. (2015). Association of ADORA1 rs2228079 and ADORA2A rs5751876 polymorphisms with gilles de la tourette syndrome in the Polish population. PloS One 10 (8), 1-13. doi: 10.1371/journal.pone.0136754

Juhasz, G., Lazary, J., Chase, D., Pegg, E., Downey, D., Toth, Z. G., et al. (2009). Variations in the cannabinoid receptor 1 gene predispose to migraine. Neurosci. Lett. 461 (2), 116-120. doi: 10.1016/j.neulet.2009.06.021

Kanaan, A. S., Jakubovski, E., and Müller-Vahl, K. (2017). Significant tic reduction in an otherwise treatment-resistant patient with gilles de la tourette syndrome following treatment with nabiximols. Brain Sci. 7 (5), 1-6. doi: 10.3390/brainsci7050047

Ketcherside, A., Noble, L. J., McIntyre, C. K., and Filbey, F. M. (2017). Cannabinoid receptor 1 gene by cannabis use interaction on cbl receptor density. Cannabis Cannabinoid Res. [Internet]. 2 (1), 202-209. doi: 10.1089/ can.2017.0007

Lazary, J., Lazary, A., Gonda, X., Benko, A., Molnar, E., Hunyady, L., et al. (2009). Promoter variants of the cannabinoid receptor 1 gene (CNR1) in interaction with 5-HTTLPR affect the anxious phenotype. Am. J. Med. Genet. Part B Neuropsychiatr. Genet. 150B (8), 1118-1127. doi: 10.1002/ajmg.b.31024

Leckman, J. F., Riddle, M. A., Berrettini, W. H., Anderson, G. M., Hardin, M., Chappell, P., et al. (1988). Elevated CSF dynorphin A [1-8] in Tourette's syndrome. Life Sci. 43 (24), 2015-2023. doi: 10.1016/0024-3205(88)90575-9

Lester, K. J., Coleman, J. R. I., Roberts, S., Keers, R., Breen, G., Bögels, S., et al. (2017). Genetic variation in the endocannabinoid system and response to cognitive behavior therapy for child anxiety disorders. Am. J. Med. Genet. Part B Neuropsychiatr. Genet. 174 (2), 144-155. doi: 10.1002/ajmg.b.32467

Mackie, K. (2006). Mechanisms of CB1 receptor signaling: endocannabinoid modulation of synaptic strength. Int. J. Obesity, S19-S23, 30. doi: 10.1038/ sj.ijo. 0803273

Martino, D., and Leckman, JF. (2013). Tourette syndrome. [Internet]. Tourette syndrome. Available from: http://ovidsp.ovid.com/ovidweb.cgi? $\mathrm{T}=\mathrm{JS} \& \mathrm{PAGE}=\mathrm{reference} \& \mathrm{D}=\mathrm{psyc} 10 \& \mathrm{NEWS}=\mathrm{N} \& A \mathrm{~N}=2013-24889-000$.

Martiny, K. (2017). Novel augmentation strategies in major depression. Danish Med. J. 64, 1-31.

Mcmahon, W. M., Van De Wetering, B. J. M., Filloux, F., Betit, K., Coon, H., and Leppert, M. (1996). Bilineal transmission and phenotypic variation of Tourette's disorder in a large pedigree. J. Am. Acad. Child Adolesc. Psychiatry 35 (5), 672-680. doi: 10.1097/00004583-199605000-00023

Mechoulam, R., and Parker, L. A. (2013). The endocannabinoid system and the brain Annu. Rev. Psychol. 64 (1), 21-47. doi: 10.1146/annurev-psych-113011-143739

Miller, S. A., Dykes, D. D., and Polesky, H. F. (1988). A simple salting out procedure for extracting DNA from human nucleated cells. Nucleic Acids Res. 16, 1215. doi: 10.1093/nar/16.3.1215

Mitjans, M., Serretti, A., Fabbri, C., Gastó, C., Catalán, R., Fañanás, L., et al. (2013). Screening genetic variability at the CNR1 gene in both major depression 
etiology and clinical response to citalopram treatment. Psychopharmacol. (Berl). 227 (3), 509-519. doi: 10.1007/s00213-013-2995-y

Müller, T. D., Reichwald, K., Wermter, A. K., Brönner, G., Nguyen, T. T., Friedel, S., et al. (2007). No evidence for an involvement of variants in the cannabinoid receptor gene (CNR1) in obesity in German children and adolescents. Mol. Genet. Metab. 90 (4), 429-434. doi: 10.1016/j.ymgme.2007.01.002

Müller, T. D., Reichwald, K., Brönner, G., Kirschner, J., Nguyen, T. T., Scherag, A., et al. (2008). Lack of association of genetic variants in genes of the endocannabinoid system with anorexia nervosa. Child Adolesc. Psychiatry Ment. Health. 2 (33), 1-7. doi: 10.1186/1753-2000-2-33

Muller-Vahl, K. R. K. (1998). Cannabinoids: possible role in patho-physiology and therapy of Gilles de la Tourette syndrome. Acta Psychiatr. Scand. 98, 502-506. doi: 10.1111/j.1600-0447.1998.tb10127.x

Muller-Vahl, K., Schneider, U., Kolbe, H., and Emrich, H. M. (1999). Treatment of Tourette's syndrome with delta-9-tetrahydrocannabinol [3]. Am. J. Psychiatry 156, 495.

Muller-Vahl, K. R. S. (2002). Combined treatment of Tourette syndrome with 9THC and dopamine receptor antagonists. J. Cannabis Ther. 2, 145-154. doi: 10.1300/J175v02n03_10

Müller-Vahl, K. R., Schneider, U., Koblenz, A., Jöbges, M., Kolbe, H., Daldrup, T., et al. (2002). Treatment of Tourette's syndrome with Delta 9-tetrahydrocannabinol (THC): a randomized crossover trial. Pharmacopsychiatry 35 (2), 57-61. doi: $10.1055 / \mathrm{s}-2002-25028$

Müller-Vahl, K. R., Schneider, U., Prevedel, H., Theloe, K., Kolbe, H., Daldrup, T., et al. (2003). ??9-tetrahydrocannabinol (THC) is effective in the treatment of tics in Tourette syndrome: a 6-week randomized trial. J. Clin. Psychiatry 64 (4), 459-465. doi: 10.4088/JCP.v64n0417

Paschou, P., Hoekstra, P. J., and Heiman, G. A. (2014). "Genetics of Tourette Syndrome," in Movement Disorders: Genetics and Models, 2nd ed. (Academic Press), 713-731.

Pertwee, R. G. (2006). The pharmacology of cannabinoid receptors and their ligands: an overview. Int. J. Obesity 30, S13-S18. doi: 10.1038/sj.ijo.0803272

Ruiz-Contreras, A. E., Román-López, T. V., Caballero-Sánchez, U., Rosas-Escobar, C. B., Ortega-Mora, E. I., Barrera-Tlapa, M. A., et al. (2017). Because difficulty is not the same for everyone: the impact of complexity in working memory is associated with cannabinoid 1 receptor genetic variation in young adults. Memory 25 (3), 335-343. doi: 10.1080/09658211.2016.1172642

Russo, E. B. (2013). Cannabis and Cannabinoids: pharmacology, toxicology, and therapeutic potential (Google eBook) [Internet]. Rev. Ther. Effects., 478. doi: 10.3389/fphar.2016.00309

Sadeghian, M., Rahmani, S., and Mansoori, A. (2018). G1359A variant of the cannabinoid receptor gene (rs1049353) and obesity-related traits and related endophenotypes: a meta-analysis. Ann. Nutr. Metab. 73 (1), 76-85. doi: 10.1159/000490668

Sandyk, R., and Bamford, C. R. (1987). Tourette syndrome. Pediatr. Neurol. 3 (1), 63. doi: 10.1016/0887-8994(87)90061-0

Sandyk, R. (1988). Self-mutilation in Tourette's syndrome. J. Child Neurol. 3, 147148. doi: 10.1177/088307388800300215

Singer, H. S., Tune, L. E., Butler, I. J., Zaczek, R., and Coyle, J. T. (1982). Clinical symptomatology, CSF neurotransmitter metabolites, and serum haloperidol levels in Tourette syndrome. Adv. Neurol. 35, 177-183.

Steiner, H., and Gerfen, C. R. (1998). "Role of dynorphin and enkephalin in the regulation of striatal output pathways and behavior," in Experimental Brain Research. (Springer), 60-76.
Suárez-Pinilla, P., Roiz-Santiañez, R., Ortiz-García de la Foz, V., Guest, P. C., Ayesa-Arriola, R., Córdova-Palomera, A., et al. (2015). Brain structural and clinical changes after first episode psychosis: focus on cannabinoid receptor 1 polymorphisms. Psychiatry Res. - Neuroimaging 233 (2), 112-119. doi: 10.1016/j.pscychresns.2015.05.005

Trainor, D., Evans, L., and Bird, R. (2016). Severe motor and vocal tics controlled with Sativex®. Australas. Psychiatry 24 (6), 541-544. doi: 10.1177/ 1039856216663737

Varadé, J., Comabella, M., Ortiz, M. A., Arroyo, R., Fernández, O., Pinto-Medel, M. J., et al. (2012). Replication study of 10 genes showing evidence for association with multiple sclerosis: validation of TMEM39A, IL12B and CLBL genes. Mult. Scler. J. 18 (7), 959-965. doi: 10.1177/1352458511432741

Wang, S., Mandell, J. D., Kumar, Y., Sun, N., Morris, M. T., Arbelaez, J., et al. (2018). De Novo sequence and copy number variants are strongly associated with tourette disorder and implicate cell polarity in pathogenesis. Cell Rep. 24 (13), 3441-3454.e12. doi: 10.1016/j.celrep.2018.08.082

Wasilewski, A., Lewandowska, U., Mosinska, P., Watala, C., Storr, M., Fichna, J., et al. (2017). Cannabinoid receptor type 1 and mu-Opioid receptor polymorphisms are associated with cyclic vomiting syndrome. Am. J. Gastroenterol. 12 (6), 933-939. doi: 10.1038/ajg.2017.73

Willsey, A. J., Fernandez, T. V., Yu, D., King, R. A., Dietrich, A., Xing, J., et al. (2017). De Novo coding variants are strongly associated with Tourette disorder. Neuron 94 (3), 486-499.e9. doi: 10.1016/j.neuron.2017.04.024

Wiskerke, J., van Mourik, Y., Schetters, D., Schoffelmeer, A. N. M., and Pattij, T. (2012). On the role of cannabinoid CBland??-opioid receptors in motor impulsivity. Front. Pharmacol. 3, 1-10. doi: 10.3389/fphar.2012.00108

Yao, Y., Xu, Y., Zhao, J., Ma, Y., Su, K., Yuan, W., et al. (2018). Detection of significant association between variants in cannabinoid receptor 1 gene (CNR1) and personality in African-American population. Front. Genet. 9, 19. doi: 10.3389/fgene.2018.00199

Yoon, D. Y., Gause, C. D., Leckman, J. F., and Singer, H. S. (2007). Frontal dopaminergic abnormality in Tourette syndrome: a postmortem analysis. $J$. Neurol. Sci. 255 (1-2), 50-56. doi: 10.1016/j.jns.2007.01.069

Yu, W., De Hert, M., Moons, T., Claes, S. J., Correll, C. U., and Van Winkel, R. (2013). CNR1 gene and risk of the metabolic syndrome in patients with schizophrenia. J. Clin. Psychopharmacol. 33 (2), 186-192. doi: 10.1097/ JCP.0b013e318283925e

Yuan, L., Zheng, W., Yang, Z., Deng, X., Song, Z., and Deng, H. (2018). Association of the AADAC gene and Tourette syndrome in a Han Chinese cohort. Neurosci. Lett. 666, 24-27. doi: 10.1016/j.neulet.2017.12.034

Conflict of Interest: The authors declare that the research was conducted in the absence of any commercial or financial relationships that could be construed as a potential conflict of interest.

Copyright $\odot 2020$ Szejko, Fichna, Safranow, Dziuba, Żekanowski and Janik. This is an open-access article distributed under the terms of the Creative Commons Attribution License (CC BY). The use, distribution or reproduction in other forums is permitted, provided the original author(s) and the copyright owner(s) are credited and that the original publication in this journal is cited, in accordance with accepted academic practice. No use, distribution or reproduction is permitted which does not comply with these terms. 